\title{
A Modular Surgical Robotic System for Image Guided Percutaneous Procedures
}

\author{
Dan Stoianovici Ph.D. ${ }^{1,2}$, Louis L. Whitcomb Ph.D. ${ }^{2}$, \\ James H. Anderson Ph.D. ${ }^{4}$, Russell H. Taylor Ph.D. ${ }^{3}$, and \\ Louis R. Kavoussi M.D. ${ }^{1}$
}

1 James Buchanan Brady Urological Institute, Johns Hopkins Medical Institutions

2 Department of Mechanical Engineering, Whiting School of Engineering

3 Department of Computer Science, Whiting School of Engineering

4 Department of Radiology, Johns Hopkins Medical Institutions

- Johns Hopkins University -

\begin{abstract}
This paper presents a robotic system for precise needle insertion under radiological guidance for surgical interventions and for delivery of therapy. It is extremely compact and is compatible with portable X-ray units and computer tomography scanners. The system presents a modular structure comprising a global positioning module, a miniature robotic module, and a radiolucent needle driver module. This system is the newest member of a growing family of modular surgical robots under development. The system may be operated stand-alone under joystick control making it readily adaptable to any operating room, or under full image guided computer control.
\end{abstract}

\section{Introduction}

Needle access required for percutaneous surgery is presently preformed in the operating room by manually inserting the needle under single-view fluoroscopic radiological guidance. This procedure is challenging; it requires extensive experience due to the lack of three-dimensional information of the inter-operative $\mathrm{X}$-ray imager.

To overcome this problem several researchers investigated the use of robotic systems to assist in needle placement. Potamianos and Davies [3], [4] proposed a stereo-pair of two x-ray views registered to a common fiducial system with a five degree of freedom (DOF) passive linkage equipped with position encoders to position a passive needle guide. Bzostek et al. [1] used an active robot (LARS [8]) for similar purposes. These systems successfully addressed difficult issues of image-to-robot registration and provided convenient means for defining target anatomy. In their present state of development, however, these robotic systems are expensive and their size makes them cumbersome for routine use in the operating room.

In contrast, our group recently reported the development of a simple, noncomputerized system, PAKY [5], which based on a minimal approach offered 
immediate application in the operation room. PAKY is a radiolucent needle driver actuated by an electrical motor. A passive arm connected to the operating table supports the driver. The surgeon uses the usual technique of "Superimposed Needle Registration" [5] to manually orient the driver and therefore the needle. PAKY is then locked into the desired orientation and needle insertion is manually controlled from a joystick. The records of twelve patients who underwent percutaneous nephrolithotomy using PAKY demonstrated that the calyx chosen by the surgeon was accessed on the first attempt in each case [2].

The PAKY device was the first member of a growing family of surgical robotic modules under development at Johns Hopkins University. This paper reports on the second member of this family, a remote center of motion (RCM) actuator module called the "MINI-RCM" and its integration and operation with the PAKY module.

In the attempt to simplify the orientation procedure, increase accuracy, reduce radiation exposure, and achieve PAKY's compatibility with advanced imaging equipment (i.e. computerized tomography) our group developed the MINIRCM. This module integrates both with PAKY and with a variety of additional end effectors presently under development. Hardware development of the MINI$\mathrm{RCM}$ is completed and pre-clinical testing of the system is in progress. A general presentation of this new system, MINI-RCM \& PAKY, is outlined next.

\section{Robotic Design}

A schematic of the system MINI-RCM \& PAKY for radiological needle insertion is presented in Figure 1. This modular system comprises the needle driver PAKY, a low dof robot (MINI-RCM), and a passive arm.

The trocar needle used for percutaneous procedures is loaded into the needle driver PAKY. The needle driver presents a radiolucent construction providing unimpeded X-ray imaging of the anatomical target. An electrical motor integrated into the driver's fixture provides automated needle insertion. The driver is constructed of acrylic, making it inexpensive to manufacture as a sterile disposable part [6].

The MINI-RCM is an extremely compact robot utilizing the RCM principle of the LARS robot reported by Taylor et al [8]. In contrast to LARS, the MINIRCM employs a chain transmission rather than a parallel linkage. This provides unrestricted rotations about the RCM point, uniform rigidity of the mechanism, and eliminates singular points. In order to accommodate different end-effectors, the MINI-RCM includes an adjustment of the location of the RCM point. Due to this adjustment the two axes of rotation ( $\mathrm{R} 1$ about $\mathrm{y}$ direction and $\mathrm{R} 2$ about $\mathrm{x}$ direction, Figure 1) may be non-orthogonal ( $79 \mathrm{deg}$ to $90 \mathrm{deg}$ ). This special scheme renders a miniaturized RCM design: the robot may be folded into a $171 \times 69 \times 52 \mathrm{~mm}$ box and it weighs only $1.6 \mathrm{Kg}$.

The needle is initially placed into the driver PAKY such that its tip is located at the remote center of motion of the MINI-RCM (RCM Point in Figure 1). For this purpose PAKY is equipped with a visible laser diode whose ray (Laser 


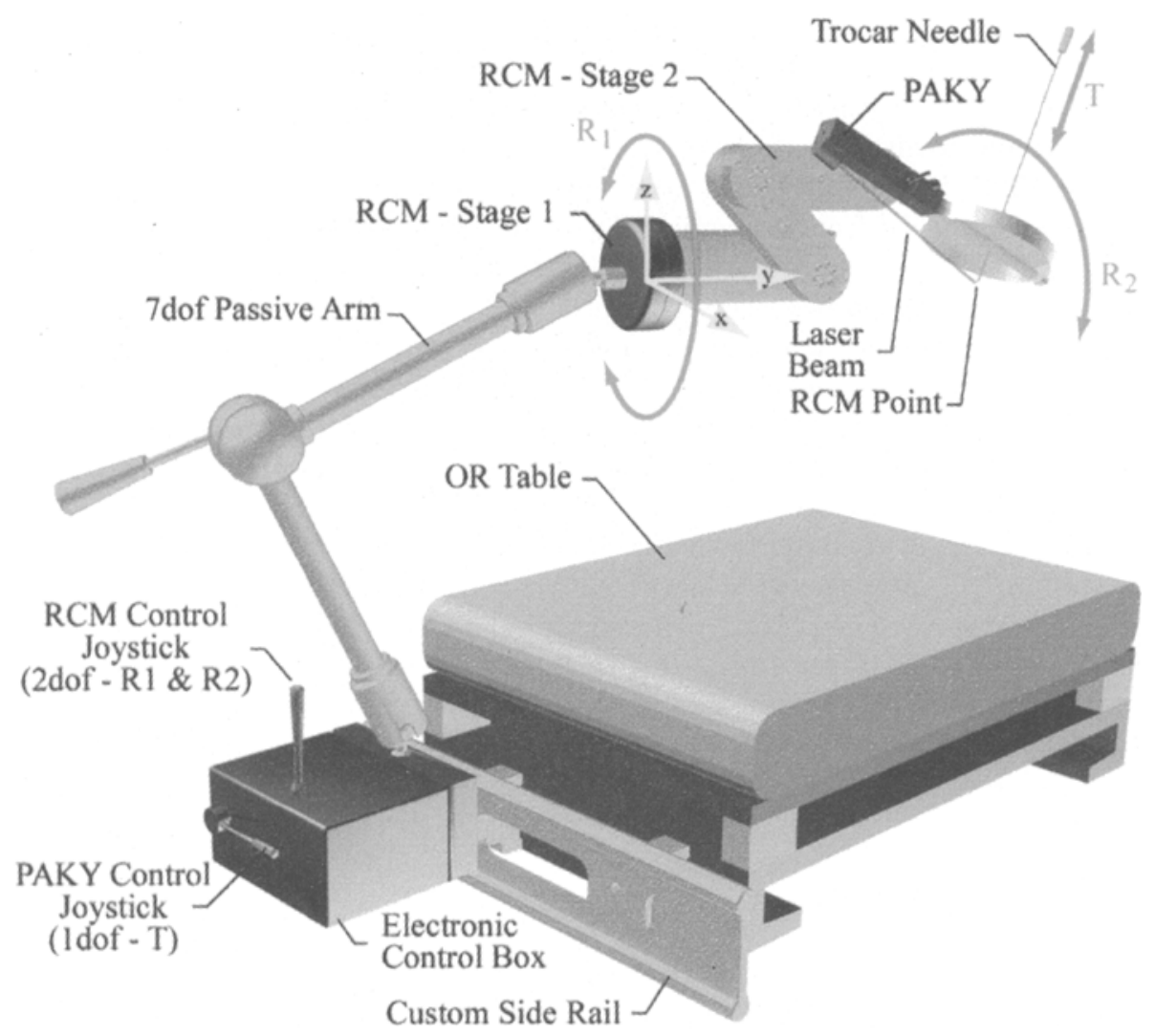

Fig. 1. Schematic of the robotic system

Beam in Figure 1) intersects the needle at the RCM point, thus helping the surgeon position the needle into the driver. The robot presents two motorized DOF implementing the rotations R1 and R2 about the RCM point (needlepoint).At present, the PAKY \& MINI-RCM robotic assembly is supported by a passive 7 DOF passive arm which may be locked at the desired position by depressing a lever. A custom rigid rail is mounted on the side of the operating room fluoroscopic table (Figure 1) to provide a sturdy base for the robotic arm. This is critical in order to provide a fixed reference frame for the robotic system and maintain needle trajectory under the insertion force.

A photograph of this modular system presenting the PAKY needle driver supported into the MINI-RCM robot is presented in Figure 2. The laser beam used for needle positioning may be observed at its point. 


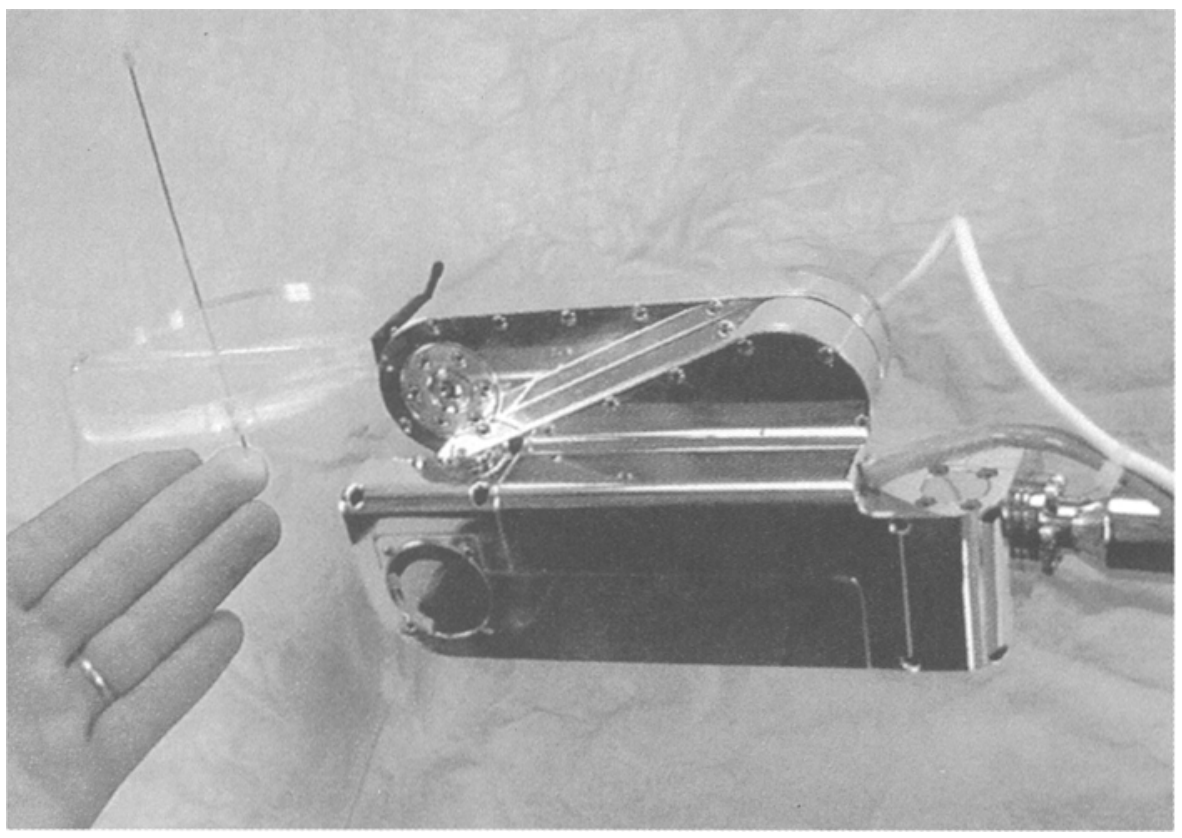

Fig. 2. MINI-RCM \& PAKY robot

\section{Operation Principle}

Inserting a needle at an arbitrary location requires six DOF. If the skin insertion site of the needle is prescribed, however, one may observe that only two rotations are necessary in order to orient the needle and only one translation is necessary to insert it. Therefore, a total number of three DOF are necessary and sufficient to aim any anatomical target while initially positioning the needle tip at the desired skin entry point.

The proposed robotic system is used as follows: Using the laser beam mark the surgeon initially positions the needle into the driver such that its tip is located at the RCM point. Then, the surgeon chooses the skin entry site and using the passive arm he/she manipulates the system such that the needlepoint is located as desired while the orientation of the needle is arbitrary. The system is locked by depressing the lever of the positioning arm. This operation does not require $\mathrm{X}$-ray imaging.

The two-rotational RCM stage is then employed to precisely orient the needle such that its axis extends into the desired target, based on radiological data. During this orientation stage the tip of the needle pivots about the skin entry point, the RCM point. When accurately oriented the needle insertion is performed using the needle driver PAKY.

The procedure may be performed in two modes: manual or automatic.

The manual mode is well suited for immediate utilization of the device in surgical procedures: it uses the portable $\mathrm{x}$-ray fluoroscopy machine (C-Arm) 


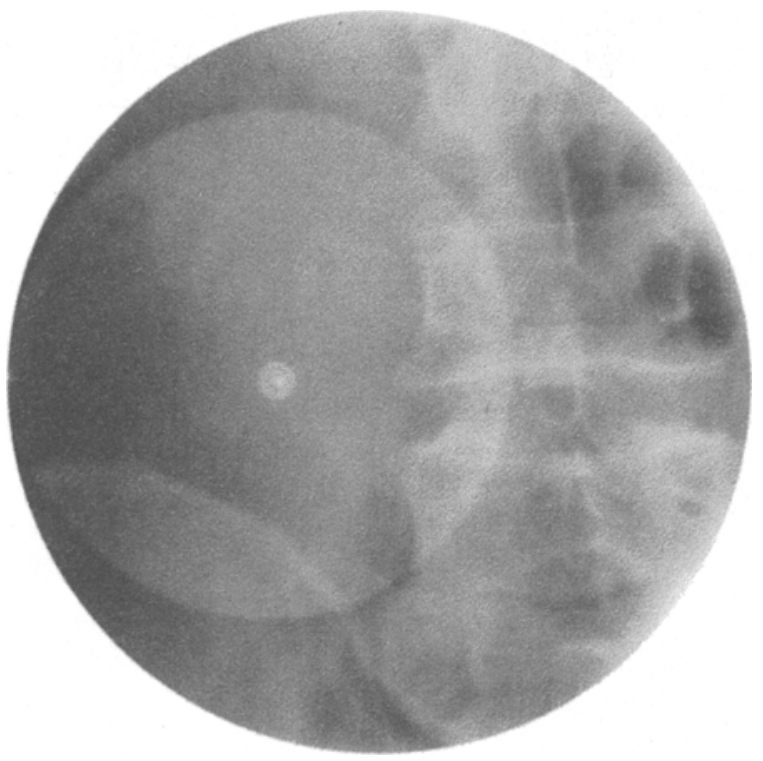

Fig. 3. PAKY's needle superimposed over a calyx of the kidney

available in the operating room, does not require additional computers or personnel, and represents an extension of the current surgical practice while increasing performance and safety. This approach employs a two-axis joystick controlling the RCM and a one-axis joystick controlling needle insertion (R1 \& R2, T in Figure 1). A velocity control loop regulates the speed of the motors by using velocity feedback obtained from the optical encoder position signals. In this operation mode the surgeon uses "Superimposed Needle Registration" [5] to orient the needle: from the joystick the surgeon orients the needle such that in fluoroscopy it appears as a single point superimposed over the desired anatomical target (Figure 3). Then, imaging from a lateral view, needle insertion is controlled from PAKY's joystick under continuos monitoring. The RCM provides fine adjustment of needle orientation increasing accuracy and reducing radiation exposure for the patient [7]. Concurrently, the joystick control of the system separates the surgeon's hands from the radiation field.

PAKY's radiolucency, the low profile of the RCM robot, and the maneuverability of the gross positioning arm are features that make this system compatible with advanced imaging devices such as computerized tomography scanners. The proposed system is used in conjunction with such a complex imager to automatically perform needle orientation and insertion based on complex imaging and registration techniques, which are subject of future development.

Clinical testing for the evaluation of the MINI-RCM \& PAKY has been commenced at the Johns Hopkins Hospital. The robot was controlled manually using the joysticks for needle orientation and insertion. X-ray fluoroscopy from a C-Arm was used. Initial observations revealed the ease of accurate needle 


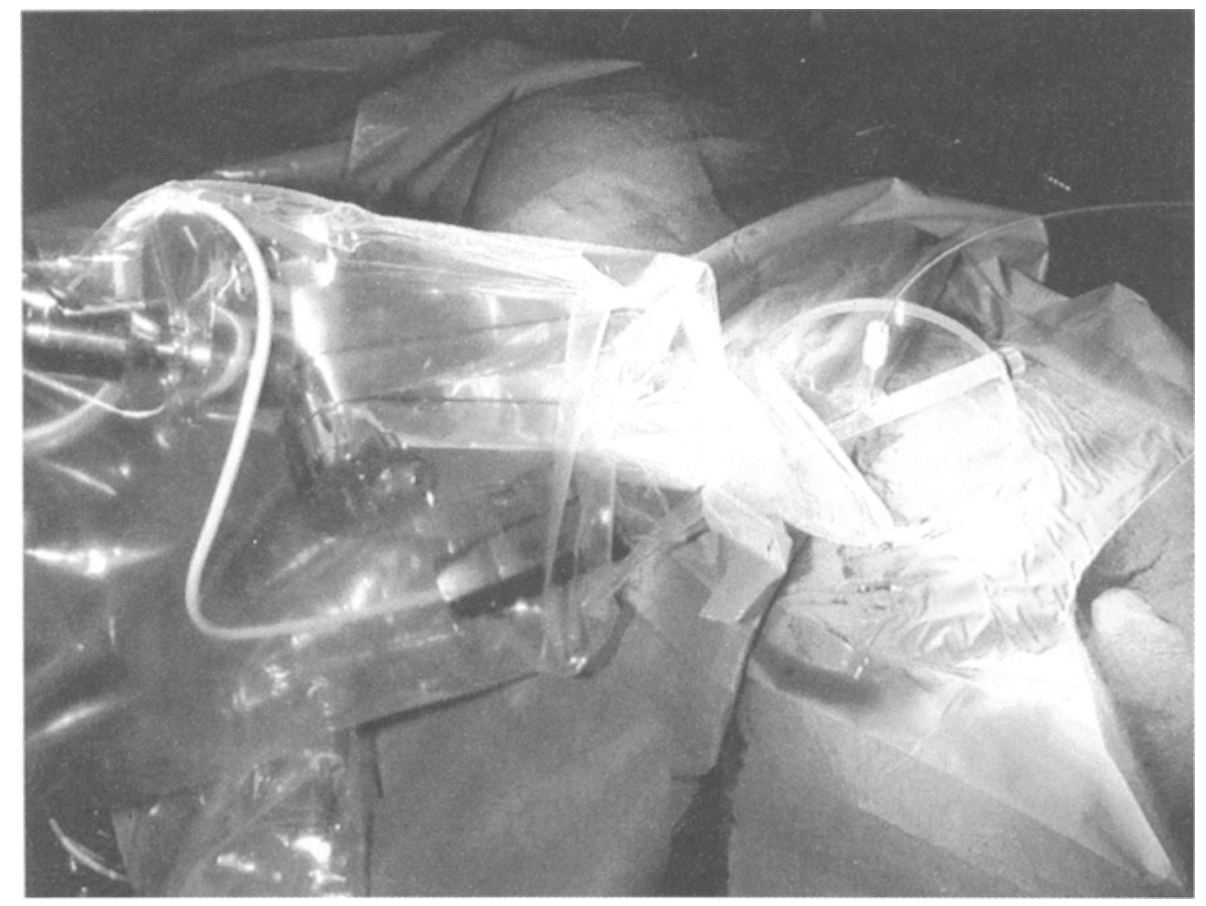

Fig. 4. MINI-RCM \& PAKY in clinical use

alignment as compared to the stand-alone PAKY system. A photograph of the MINI-RCM \& PAKY performing a percutaneous access procedure is presented in Figure 4. The needle is placed into the collecting system of the kidney and an access wire is passed through.

\section{Safety Features}

Safety is essential for surgical robots. The proposed system addresses safety by employing a low DOF robot, by decoupling needle orientation from the needle insertion, and by using non-backdrivable transmissions.

As previously presented, percutaneous needle access implies only 3 DOF. Our system implements all and only these DOF, such that the system has a minimal architecture and restricts arbitrary movements.

Furthermore, needle orientation and insertion are implemented by different mechanisms, which are independently activated by safety buttons on the joysticks. For needle alignment the surgeon activates only the MINI-RCM and orients the needle using the two-dimensional joystick while the needle pivots around the skin insertion site. When properly aligned, the RCM is deactivated. Needle insertion is then enabled by activating PAKY. Using this scheme, the system prevents the needle to be inserted before being properly aligned and prevents changes of orientation while inserting it. 
In addition, the robot uses worm transmissions rendering a non-backdrivable mechanism. This preserves robot's configuration when deactivated or in the event of a power failure.

\section{Conclusion}

The proposed system extends PAKY's performance and capabilities for performing image-guided needle access by employing a low DOF RCM robot. The particular design of the RCM robot renders miniaturization and versatility making this system fully compatible with complex imaging equipment. Concurrently, the system integrates a manual operation mode such that the system may be rapidly transferred to the surgical setting.

\section{References}

1. Bzostek, A., Schreiner, S., Barnes, A.C., Cadeddu, J.A., Roberts, W., Anderson, J.H., Taylor, R.H., Kavoussi, L.R.: An automated system for precise percutaneous access of the renal collecting system. Lecture Notes in Computer Science, Vol. 1205. SpringerVerlag, Berlin Heidelberg New York (1997) 299-308

2. Cadeddu J.A., Stoianovici D., Chen R.N., Moore R.G., Kavoussi L.R.: Stereotactic mechanical percutaneous renal access. Journal of Endourology, Vol. 12, No. 2, (1998) $121-126$

3. Potamianos, P., Davies, B.L., and Hibberd, R.D.: Intra-operative imaging guidance for keyhole surgery methodology and calibration. Proc. First Int. Symposium on Medical Robotics and Computer Assisted Surgery, Pittsburgh, PA. (1994) 98-104

4. Potamianos, P., Davies, B.L., and Hibberd, R.D.: Intra-operative registration for percutaneous surgery. Proc. First Int. Symposium on Medical Robotics and Computer Assisted Surgery, Baltimore, MD. (1995) 156-164

5. Stoianovici, D., Cadeddu, J., A., Demaree, R., D., Basile, H., A., Taylor, R., H., Whitcomb, L., L., Sharpe, W. N. Jr., Kavoussi, L., R.: An Efficient Needle Injection Technique and Radiological Guidance Method for Percutaneous Procedures. Lecture Notes in Computer Science, Vol. 1205. Springer-Verlag, Berlin Heidelberg New York (1997) 295-298

6. Stoianovici, D., Cadeddu, J., A., Demaree, R., D., Basile, H., A., Taylor, R., H., Whitcomb, L., L., Kavoussi, L., R.: A Novel Mechanical Transmission Applied to Percutaneous Renal Access. Proceedings of the ASME Dynamic Systems and Control Division, DSC, Vol. 61 (1997) 401-406

7. Stoianovici, D., Cadeddu, J., A., Whitcomb, L., L., Taylor, R., H., Kavoussi, L., R.: A Robotic System for Precise Percutaneous Needle Insertion. Thirteen Annual Meeting of the Society for Urology and Engineering, May 1998, San Diego, CA (1998) 5-6

8. Taylor R.H., Funda J., Eldridge B., Gruben K., LaRose D., Gomory S., Talamini M., Kavoussi L.R., Anderson J.: A Telerobotic Assistant for Laparoscopic Surgery. IEEE Engineering in Medicine and Biology Magazine, Vol. 14, (1995) 279-287 